ESJ Social Sciences

\title{
Economie d'échelle à l'interne et Covid-19 : Une tentative de voie de sortie en RDC
}

\begin{abstract}
Tsongo Kayiri Jean de Dieu
Doctorant, Centre d'Etude et de Recherche en Economie et Gestion (CEREG), Faculté des Sciences Economiques et de Gestion (FSEG) de l'Université de Yaoundé II, Cameroun
\end{abstract}

Shiko'a Abwe André

Indépendant, Licencié en Sciences Commerciales et Financières: Douanes et Commerce extérieur, Institut Supérieur de Commerce, ISC-Goma

Doi:10.19044/esj.2021.v17n12p210

Submitted: 01 March 2021

Accepted: 05 April 2021

Published: 30 April 2021
Copyright 2021 Author(s)

Under Creative Commons BY-NC-ND

4.0 OPEN

ACCESS

Cite As:

Tsongo, KJ., and Shiko, AA. (2021). Economie d'échelle à l'interne et Covid-19: Une tentative de voie de sortie en RDC. European Scientific Journal, ESJ, 17(12), 210. https://doi.org/10.19044/esj.2021.v17n12p210

Résumé:

Les pays en développement sont soumis à des études approfondies pour s'adapter à une nouvelle configuration économique naturelle à la suite de la situation d'autarcie imposée par la Covid-19. Cet article analyse la possibilité qui puisse exister par application d'une économie d'échelle interne pour faire face aux effets économiques de la Covid-19 en République Démocratique du Congo (RDC). La comparaison des variances a été faite à partir du test $\mathrm{F}$ de Fisher-Snedecor. N'étant pas à confondre au test exact de Fisher, cette loi a servi pour tester la participation de secteurs économiques dans le Produit Intérieur Brut (PIB) afin d'opter le secteur à appliquer cette économie. Les résultats font étant de l'absence de différence de moyennes de secteurs économiques, ce qui dit qu'aucun secteur ne contribue significativement à la production du pays. Pratiquement, les résultats invitent les autorités politiques et opérateurs économiques à l'application de l'économie d'échelle dans un secteur de choix. Mais, si on se contentait des paramètres de tendance centrale et de modèle Solow-Swan, il serait conseillé d'exploiter plus le secteur primaire accompagné du secteur tertiaire. De cette recherche, on peut noter la présence d'une limite sur la 
détermination des facteurs de production d'ordre général qui peuvent se déplacer entre les secteurs et des facteurs spécifiques.

Keywords: Economie- d'échelle, autarcie-forcée, secteurs économiques, COVID-19, RDC.

\title{
Internal Economy of Scale and Covid-19: An Attempt at an Exit Route in The DRC
}

\author{
Tsongo Kayiri Jean de Dieu \\ Doctorant, Centre d'Etude et de Recherche en Economie et Gestion (CEREG), Faculté des \\ Sciences Economiques et de Gestion (FSEG) de l'Université de Yaoundé II, Cameroun
}

\section{Shiko'a Abwe André}

Indépendant, Licencié en Sciences Commerciales et Financières: Douanes et Commerce extérieur, Institut Supérieur de Commerce, ISC-Goma

\begin{abstract}
:
Developing countries are undergoing extensive studies to adapt to a new natural economic configuration as a result of the autarky imposed by Covid-19. This paper analyzes the possibility of applying an internal economy of scale to cope with the economic effects of Covid-19 in the Democratic Republic of Congo (DRC). The comparison of variances was done using the Fisher-Snedecor F test. Not to be confused with Fisher's exact test, this law was used to test the participation of economic sectors in the Gross Domestic Product (GDP) in order to choose the sector to apply this economy. The results show that there is no difference in the averages of the economic sectors, which means that no sector contributes significantly to the production of the country. Practically, the results invite the political authorities and economic operators to apply the economy of scale in a sector of choice. However, if we were content with the parameters of the central tendency and the Solow-Swan model, it would be advisable to exploit the primary sector along with the tertiary sector. From this research, we can note the presence of a limit on the determination of general factors of production that can move between sectors and specific factors.
\end{abstract}

Keywords: Economy-of-scale, autarky-forced, economic sectors, COVID19, DRC. 


\section{Introduction}

La situation économique mondiale continue de subir les séquences de la crise économique et financière de 2009. Egalement, la croissance a légèrement diminué en 2013 aux Etats-Unis se situant à 2,2\% contre 2,3\% en 2012, dans le pays de la zone Euro, après une baisse de 0,7\% l'année 2013 (ANSD $\left.{ }^{1}, 2015\right)$. Or, les pays font face à des perturbations de leurs prévisions depuis l'arrivée de la pandémie à COVID-19.

La crise Covid a eu des incidences financières redoutables. L'accroissement de l'endettement des entreprises aux Etats-Unis s'est redoublé d'une détérioration de sa qualité, à la fois dans l'univers investment grade et dans l'univers high yield. Dans de nombreux pays émergents et en développement, la situation est devenue critique (Michel \& Sabrina, 2020).

Les crises systémiques, dont le moment de basculement (moment Minsky) est la panique pour la liquidité, sont gérées par les banques centrales par la prise en charge du marché monétaire, d'une part, le sauvetage des banques et autres institutions financières, d'autre part (BRI, 2020).

En mars 2020, quand le monde prit conscience de la gravité de la pandémie et quand les premiers confinements furent décidés, il n'allait pas de soi que les banques centrales devaient monter en première ligne. Cette crise était très différente des précédentes. La réponse des gouvernements a été rapide et massive : $11700 \mathrm{Md} \$$ ou $12 \%$ du PIB mondial selon l'estimation du Fonds monétaire international (FMI) en septembre 2020, dont la moitié sous forme de dépenses supplémentaires, de baisses d'impôt et de reports de charge et l'autre moitié sous forme de garanties et de prises de participation. (Benoit, 2020).

En RDC, par exemple, l'Institut d'émission a décidé, en date du 24 mars 2020, d'assouplir sa politique monétaire. Cette décision visait à garantir la liquidité au système bancaire et l'économie et procéder à une baisse de son taux directeur de $9,0 \%$ à $7,5 \%$ ainsi qu'à la réduction du coefficient de la réserve obligatoire sur les dépôt à vue en monnaie locale, lequel est passé de $2,0 \%$ à $0,0 \%$ (https://Deskeco.com).

Globalement, la croissance économique est ressortie à 3,3\% en 2013, après 3,4\% en 2012 (ANSD, 2015) mais au Benin, la contribution importante et grandissante de secteurs primaires et tertiaires à la croissance du PIB reflète l'impact de la production cantonnière et du commerce portuaire (Touré \& Macwilliam, 2014). Pendant que trente ans plus tard, nous constatons que les habitants des pays en développement dont la subsistance est tributaire de l'agriculture sont encore, en règle générale, bien plus

\footnotetext{
${ }^{1}$ ANSD : est une Agence Nationale de la Statistique et de la Démographie en République du Sénégal. Elle est sous tutelle du Ministère de l'économie et des finances.
} 
pauvres que ceux qui travaillent dans d'autres secteurs de l'économie et qu'ils représentent en forte proportion (Cervantes-Godey \& Dewbre, 2010).

Quel secteur économique doit-on concentrer les activités économiques pour en tirer une économie d'échelle interne en RDC?

Bresciani et Valdés font en particulier ressortir que la contribution de l'agriculture à la réduction de la pauvreté est systématiquement supérieure à la part de l'agriculture dans le PIB (Cervantes-Godey \& Dewbre, 2010). Cependant, le contexte diffère selon le pays, le secteur des services représente aujourd'hui plus de $70 \%$ de l'emploi total et de la valeur ajoutée dans les économies de l'OCDE (OCDE, 2005).

$\mathrm{Au}$ cours de trente dernières années, dans l'économie du Québec, la part de l'emploi dans les services passe de 56,9\% en 1966 à 73,5\% en 1995, soit une progression de $16,6 \%$. Cette progression s'est effectuée aux dépens du secteur primaire, dont la part décroit de $8,2 \%$ à $3,5 \%$ et du secteur secondaire (de 34,9\% à 23,0\%) (Direction de l'analyse de la Conjoncture industrielle, 1996). La croissance économique en 2019 a été principalement impulsée par le secteur tertiaire à hauteur de $1,7 \%$ contrairement à 2018 où le taux de croissance de $0,89 \%$ a été plus impacté par le secteur primaire (Banque Centrale du Congo, 2019).

Cet article démontrera statiquement le secteur qui participe différemment aux autres afin de voir si la spécialisation de ce secteur pourrait permettre à la République Démocratique du Congo de faire face aux aléas économiques causés par la Covid-19.

\section{DEVELOPPEMENT THEORIQUE}

Dans les théories préindustrielles, la croissance est expliquée par la progression de la force de travail, soit par la démographie et les évolutions sociétales. Selon Malthus (1798) cité par Donald (2007), si elle n'est pas freinée, la population s'accroit en progression géométrique. Les subsistances ne s'accroissent qu'en progression arithmétique. Donald (2007), d'où, il tient de limiter les naissances. Pierre \& Annan (2005) démontrent l'importance du capital humain ou physique à long terme. L'offre (le capital, la terre, ...) a une importance par rapport aux facteurs relatifs à la demande (prix modélisés par les politiques internes et externes). Ces auteurs finissent par montrer que dans une phase de développement, les politiques internes, avant des taxes sur le secteur agricole permettent le transfert de ressources vers les autres secteurs.

Par contre, d'autres économistes expliquent la croissance par l'accumulation du capital, on parle des théories de l'accumulation du capital productif en économie fermée. Commençons par ceux (vers les années 19501980) qui estiment que la croissance est expliquée par des facteurs exogènes. Pour Harrod et Domar cités par Kibala (2019), une croissance stable ou 
équilibrée exige à la fois le plein emploi et l'équilibre entre l'épargne et l'investissement (fil de rasoir) ; elle nécessite aussi une combinaison optimale des facteurs travail et capital considérés complémentaires. Ces auteurs estiment que la croissance n'est pas stable - soit que l'équilibre de plein emploi est un heureux hasard - du fait de l'insuffisance de capital, et parce qu'il n'est pas évident d'observer ces équilibres (équilibre épargne et équilibre emploi).

Autrement dit, pour Harrod et Domar, l'épargne est le moteur de la croissance, et les politiques d'encouragement à l'épargne ou à l'investissement sont préconisées. Contrairement à ces auteurs, Solow et Swan (1956) cités par Kibala (2019), on parle de modèle de Solow-Swan. Ils refusent l'idée d'un « fil de rasoir » et considèrent que si les marchés sont en équilibre, les facteurs de production sont substituables et ont chacun un rendement marginal décroissant, mais la production a des rendements d'échelle constants.

Les facteurs mobiles, qui peuvent passer d'un secteur à un autre, sont dans une situation intermédiaire : ils peuvent aussi bien gagner que perdre à l'ouverture (Krugman \& al., 2017). Si Krugman tire conclusion sur les effets de l'ouverture de l'économie, cet article veut déterminer seulement le secteur qui profiterait de l'économie d'échelle par l'appui du gouvernement selon sa contribution dans la production du pays.

Solow-Swan estiment que - sous l'hypothèse de rendement marginal décroissant de capital - les effets positifs de l'épargne sur la croissance sont transitoires et finissent par s'épuiser à long terme quand l'économie atteint l'état stationnaire ou l'équilibre ; à ce niveau, le PIB par tête dépend du taux d'épargne, du taux de croissance démographique et du taux de dépréciation du capital (Mankiw, Romer et Weil, 1992). On parle de modèle de Solow augmenté. Après avoir confronté le modèle de Solow-swan aux faits, ils présentent le " capital humain » (investissement) comme un facteur de croissance autant que le capital physique. L'idée est que la formation (dépenses en éducation) améliore les capacités productives des individus.

La prise en compte du capital humain a permis à ces auteurs de valider empiriquement les thèses de Solow-Swan (convergence économique). L'implication de l'individu au travail est un élément important comme le montrent Allen \& Meyer cités par Mohamed \& al. (2016) que l'implication est un état psychologique comportant une dimension affective, une dimension calculée et une dimension normative, et ayant une influence sur la décision de rester membre de l'organisation. Mohamed \& al. concluent que la participation des salariés dans la prise de décision est un élément qui permet de développer l'implication.

Bien d'autres économistes (vers les années 1980-1990) pensent autrement, soutenant que la croissance est expliquée par des facteurs 
endogènes, et s'intéressent aux déterminants de la productivité globale des facteurs/PGF (la technologie). On parle des théories de croissance endogène qui stipulent que, en l'absence de progrès technique exogène, la croissance peut perdurer sous l'hypothèse des rendements croissants du capital au niveau macroéconomique. Selon Romer (1986), en l'absence de progrès technique, la croissance est autoentretenue grâce aux externalités positives (externalité de réseau, accumulation d'un ensemble de connaissances et savoir-faire, etc.).

Ici, les politiques publiques doivent coordonner les décisions privées pour faire jouer les externalités. Pour Barro (1990), le capital public ou les infrastructures publiques (dépenses publiques : éducation, services publics, ...) empêchent la baisse des rendements marginaux du capital privé et favorisent la croissance à long terme par un effet d'offre (et non de demande). Les études empiriques de l'OCDE (Kibala, 2019) confirment cette thèse.

Toutefois, l'on note que le capital public peut freiner la croissance si son coût d'opportunité (distorsion, effets de congestion) est très élevé (l'impôt réduit la rentabilité des investissements et freinent ainsi l'accumulation du capital privé). Aghion \& Howitt (1992) présentent « l'innovation " (les gains attendus) comme un facteur de croissance. Ils estiment que les gains et effort de l'innovation (soit l'extension de la gamme de produits ou différenciation horizontale, soit l'amélioration de la qualité des produits ou différenciation verticale ou d'échelles de qualité à la manière de Schumpeter) sont fonction du degré de concurrence sur le marché des produits et du régime de la protection intellectuelle.

Par contre, l'économie Congolaise a affiché une croissance de 6,5\% en 2011 contre 7,2\% en 2010, tirée par l'agriculture, les industries extraverties et le commerce (OCDE, 2012). Le manioc est la culture dominante et relativement importante ( $90 \%$ des fermes du collidor Monkoto) et constitue l'aliment de base dans la zone avec des chaines de valeur telles que la chikwangue et la farine de manioc, utile dans la préparation du fufu et d'autre part, comme source de revenu par la vente de "lotoko », l'alcool indigène (Bonkena B.P. \& al. 2000). Les institutions faibles du pays ont échoué à bâtir les fondations d'une économie résiliente et à absorber les chocs externes, exposant ainsi la société à des cycles de violence et d'appauvrissement (Banque Mondiale, 2018).

La croissance économique en RDC a été robuste, le PIB progressant en termes réels de $7 \%$ en moyenne entre 2010 et 2012, jusqu'à atteindre $8,5 \%$ en 2013 et $8,9 \%$ en 2014, dépassant la moyenne des pays de l'Afrique Subsaharienne ; la croissance est tirée par le secteur minier qui s'est accru de 10,5\% en moyenne en 2010-2013, et qui bénéficie des partenariats entre des firmes étrangères et des sociétés Congolaises (Banque Mondiale 2014). Le 
commerce a connu, une expansion de $7 \%$ en 2011. Sa contribution à la croissance est passée à 1,4 point, contre 0,99 en 2010. Il a tiré du bon comportement du secteur minier et de l'amélioration des infrastructures routières. Le secteur des transports et des communications a faiblement contribué à la croissance $(0,4$ point contre 0,3 point en 2010$)$.

En définitif, la croissance en 2011 a profité du dynamisme de la demande. La consommation (privée et publique) a contribué à 3,3 point (OCDE, 2012). Dans l'économie Hattienne, par exemple, l'agriculture régresse et est dépassée par la branche « commerce, restaurant et hôtels ». Le secteur industriel reste modeste et peu dynamique (www.brh.net/index.html).

De toutes ces études, les secteurs économiques dans plusieurs pays influencent la croissance économique durant les décennies. Cependant, le choix d'un secteur à la merci d'autre secteur engendre des coûts. Il y aura d'une part, les gagnants et les perdants, d'autre part comme le montrent Krugman P. \& al. (2017) : " le commerce international a souvent des effets importants sur la distribution des revenus au sein d'un pays. Il fait donc des perdants et des gagnants. La répartition des revenus entre les agents évolue pour deux raisons : les facteurs de production ne peuvent pas se déplacer d'un secteur à l'autre instantanément et sans aucun coût, et le mouvement de spécialisation résultant de l'ouverture commerciale n'a pas les mêmes conséquences sur la demande des différents facteurs de production ».

Devant un contexte imposé par la Covid-19, « le développement d'une collaboration gagant-gagant accompagnée d'une maitrise des risques contribuent à améliorer le niveau d'agilité et de performance de la chaîne d'approvisionnement » (Abrighach \& al. 2019) entre les pays mais ceux-ci ont opté pour les fermetures des frontières et très loin aux confinements entres les villes.

Sans moindre attention d'information sur les facteurs de production d'ordre général, qui peuvent se déplacer entre les secteurs, et des facteurs spécifiques qui ne peuvent être employés que dans les secteurs précis, cet article se limitera à voir en RDC le secteur qui pourra bénéficier de l'économie d'échelle pour contourner les aléas de la Covid-19.

Cependant, l'analyse de cette variable pourra compléter cette étude pour permettre la RDC de bien contrôler son économie comme insistent Mssassi \& al. (2018) sur les contributions de la théorie de la contingence selon lesquelles une organisation qui surveille son environnement est d'autant mieux informée dans la mesure où elle dispose d'informations de qualité suffisantes ou encore qui sont essentielles sur leur environnement. La richesse d'informations ancre bien une richesse de quantité et de qualité. 


\section{Demarche Methodologique}

La démarche méthodologique est axée sur l'approche économétrique qui consiste à analyser plusieurs variables (secteurs : primaire, secondaire et tertiaire) tout en comparant les variances. Il sera question d'identifier le secteur à encourager avec le crédit bancaire en vue de faire face aux aléas de la Covid-19. Le rapport de la Banque Centrale du Congo servira de source des données :

Tableau 1 : Contributions de différents secteurs au Produit Intérieur Brut (En millions de CDF courant)

\begin{tabular}{|c|c|c|c|c|c|c|c|c|c|c|}
\hline anch & 2009 & 2010 & 2011 & 2012 & 2013 & 2014 & 2015 & 2016 & 2017 & 2018 \\
\hline Secteur & 1751,2 & 7575356,4 & 9760200,4 & 10696781,2 & 11861980,1 & 13493927,4 & 13628921,6 & 14232480,4 & 21071943,5 & 29744921,7 \\
\hline & 3575155,9 & 4 & 776,9 & 9797,3 & 48 & 6] & 6450068,6 & 6978142,6 & 969692,2 & 14657898,9 \\
\hline Agriculture & 24000 & 4010000,3 & 47009732 & 5263806,5 & 5572388,4 & 5873449,0 & 6203304,2 & 6702671,5 & 10544749,2 & 14108549,8 \\
\hline Vi & 3013 & 3952706,7 & 4693058,9 & 52009943,7 & 5494122,5 & $57 \% 5871,2$ & 6115548,7 & 6605460,6 & 10391392,0 & 13903270,2 \\
\hline Rente & 4918 & 57279,6 & 67914,3 & 74942 & 78260 & 87577,7 & 87755,4 & 97210,9 & 153357,2 & 205279,7 \\
\hline Sylicath & 129593,3 & 169112,6 & 200295,2 & 216462,1 & 22678,1 & 203418,2 & 235524,0 & 263336,9 & 405608,8 & 523290,0 \\
\hline Eéveg & 0.2 & 7236,2 & 8508,5 & 9448,7 & 9819,9 & 10341,4 & 11240,4 & 12134,2 & 19334,2 & 26059,1 \\
\hline Indust & 356595,3 & 3385894,3 & 4790423,5 & 5186963,9 & 6057008,6 & 7326718,8 & 7178853,0 & 7254337,8 & 10102251,3 & 15087022,7 \\
\hline ctou & 3629200,1 & 4169632,9 & 4937335,4 & 5663507,3 & 6349940,3 & 6952900,9 & 7464236,0 & 8188339,2 & 13388847,9 & 10022005 \\
\hline Industri & 2850452,2 & 3169171,1 & 3649184,5 & 4140563,5 & 4623444,0 & 5167796,5 & 6014081,6 & 6852323,8 & 10849621,5 & 13648333,6 \\
\hline Agro indu & 2253737,8 & 2541111,5 & 2843741,9 & 3354043,8 & 3803700,6 & 4459077,8 & 517956,2 & 5932477,8 & 9307232,0 & 11551328,0 \\
\hline Autres int & 617714,4 & 628059,6 & 805442,6 & 77659,7 & 739603,4 & 708718,8 & 836125,4 & 919846,0 & 1542389,5 & 2097005,6 \\
\hline Baiments et Travas pu & 165301,2 & 7888000,5 & 1055675,0 & 1264563,2 & 1448504,5 & 1485564,5 & 1163849,6 & 980965,9 & 1993879,7 & 4651735,9 \\
\hline Electricité, & 613366,7 & 211581,3 & 232475,9 & 2583500,6 & 277991,7 & 29959,9 & 2065304,9 & 365049,6 & 544946,7 & 522586,4 \\
\hline Secteur: & 5646184,1 & 6740687,6 & 7673851,3 & 8730200,5 & 9513006,7 & 10296062,1 & 11364783,1 & 12399597,8 & 18328996,3 & 24332550,3 \\
\hline Commerce de & 1708909,5 & 2080030,0 & 2391107,6 & 2842133,3 & 3110794,7 & 3304900,4 & 37003175,8 & 4129905,8 & 6693143,4 & 8914351,3 \\
\hline Transpo & 1795066,9 & 2092046,8 & 23608022 & 2701538,0 & 2965169,9 & 3206974,4 & 3562392,0 & 3859941,4 & 4766172,0 & 6238320,1 \\
\hline Services & 1183699,6 & 1487205 & 1697812,7 & 1889868,8 & 2027802,5 & 2279221,3 & 2514884,1 & 2716312,2 & 4287753,1 & 5884120,1 \\
\hline Services & 1104123,0 & 1252290,6 & 1418022,8 & 1510537,8 & 1592494,0 & 1739391,0 & 1822896,9 & 1965013,0 & 3043776,0 & 3966763,2 \\
\hline SIFIM & $.145615,0$ & $-170885,0$ & $-193894,0$ & $-213797,5$ & $.2033254,4$ & $.236428,1$ & .2585668 .8 & 291574,6 & $-461848,2$ & $-671004,5$ \\
\hline PIB au co & 14207135,5 & 18485676,9 & 22371387,1 & 25090569,1 & 27724927,0 & 30742890,4 & 32457940,7 & 34820417,4 & 52769387,8 & 72900127,7 \\
\hline Drots et & 894052,4 & 1051000,0 & 1388037,5 & 1863987,9 & 2326252,4 & 2481098,07 & 2653265,3 & 2696975,0 & 28060705,4 & 3789144,3 \\
\hline PIB aux prix du marché & 15101187,9 & 19536676,9 & 23759424,6 & 26954556,9 & 30051179,4 & 33223968,5 & 35111226,0 & 37517392,4 & 55676093,1 & 76689272,0 \\
\hline
\end{tabular}

Source : Rapport de la Banque Centrale du Congo 2018.

La comparaison des variances sera faite à partir du test $\mathrm{F}$ de Fisher (ou Fisher-Snedecor), n'est pas à confondre avec test exact de Fisher qui est un test de rapport de variances avec des degrés de libertés (Nicolas, 2011).

Tableau 2: Rapport détaillé des paramètres de tendance centrale

\begin{tabular}{|l|r|r|r|r|}
\hline Groupes & \multicolumn{1}{l|}{$\begin{array}{l}\text { Nombre } \\
\text { d'échantillons }\end{array}$} & \multicolumn{1}{l|}{ Somme } & \multicolumn{1}{l|}{ Moyenne } & \multicolumn{1}{l|}{ Variance } \\
\hline Colonne 1 & 10 & 136998263,9 & 13699826,39 & 50492135989341,30 \\
\hline Colonne 2 & 10 & 79566195,8 & 7956619,58 & 22100308384497,90 \\
\hline Colonne 3 & 10 & 115025999 & 11502599,9 & 32973734709053,10 \\
\hline
\end{tabular}

Source : Généré par Ms Excel à partir de nos estimations.

Le secteur primaire a participé à une grande part dans le PIB et suivi par le secteur tertiaire durant la dernière décennie. Le tableau ci-dessous 
montre la participation de différents secteurs durant toutes les années sous étude avant de tester statistiquement :

Tableau 3 : Participation de secteurs dans le PIB

\begin{tabular}{|c|c|c|c|c|}
\hline Années \% & $\begin{array}{c}\text { Secteur } \\
\text { Primaire }\end{array}$ & Secteur secondaire & Secteur tertiaire & $\begin{array}{l}\text { PIB au coût } \\
\text { du marché }\end{array}$ \\
\hline 2009 & 4931751,20 & 3629200,10 & 5646184,10 & $\begin{array}{l}14207 \\
135,40 \\
\end{array}$ \\
\hline$\%$ & 34,71 & 25,54 & 39,74 & 100,00 \\
\hline 2010 & 7575356,40 & 4169632,90 & 6740687,60 & $\begin{array}{r}18485676,9 \\
0\end{array}$ \\
\hline$\%$ & 40,98 & 22,56 & 36,46 & 100,00 \\
\hline 2011 & 9760200,40 & 4937335,40 & 7673851,30 & $\begin{array}{r}22371387,1 \\
0 \\
\end{array}$ \\
\hline$\%$ & 43,63 & 22,07 & 34,30 & 100,00 \\
\hline 2012 & 10696781,20 & 5663507,30 & 8730280,50 & $\begin{array}{r}25090569,0 \\
0 \\
\end{array}$ \\
\hline$\%$ & 42,63 & 22,57 & 34,80 & 100,00 \\
\hline 2013 & 11861980,10 & 6349940,30 & 9513006,70 & $\begin{array}{r}27724927,1 \\
0 \\
\end{array}$ \\
\hline$\%$ & 42,78 & 22,90 & 34,31 & 100,00 \\
\hline 2014 & 13493927,40 & 6952900,90 & 10296062,10 & $\begin{array}{r}30742890,4 \\
0 \\
\end{array}$ \\
\hline$\%$ & 43,89 & 22,62 & 33,49 & 100,00 \\
\hline 2015 & 13628921,60 & 7464236,00 & 11364783,10 & $\begin{array}{r}32457940,7 \\
0 \\
\end{array}$ \\
\hline$\%$ & 41,99 & 23,00 & 35,01 & 100,00 \\
\hline 2016 & 14232480,40 & 8188339,20 & 12399597,00 & $\begin{array}{r}34820416,6 \\
0 \\
\end{array}$ \\
\hline$\%$ & 40,87 & 23,52 & 35,61 & 100,00 \\
\hline 2017 & 21071943,50 & 13388447,90 & 18328996,30 & $\begin{array}{r}52789387,7 \\
0 \\
\end{array}$ \\
\hline$\%$ & 39,92 & 25,36 & 34,72 & 100,00 \\
\hline 2018 & 29744921,70 & 18822655,80 & 24332550,30 & $\begin{array}{r}72900127,8 \\
0 \\
\end{array}$ \\
\hline$\%$ & 40,80 & 25,82 & 33,38 & 100,00 \\
\hline TOTAL & 124491156,30 & 71767362,80 & 102639127,30 & $\begin{array}{r}298897646, \\
40 \\
\end{array}$ \\
\hline Moyennes & 12449115,63 & 7176736,28 & 10263912,73 & $\begin{array}{r}29889764,6 \\
4 \\
\end{array}$ \\
\hline$\%$ & 41,65 & 24,01 & 34,34 & 100,00 \\
\hline
\end{tabular}

Source : Synthétisé à partir le rapport de BCC 2018

Sauf l'année 2009 où le secteur tertiaire a devancé d'autres secteurs, le reste d'années le secteur primaire a participé à une grande part, ce résultat rejoint celui de Bonkena B.P. \& al. (2000) selon lequel le manioc est la culture dominante et relativement importante $(90 \%$ des fermes du collidor Monkoto).

Les moyennes d'année s'élèvent respectivement à une hauteur de $41,65 \%$ pour le secteur primaire, le tertiaire quant à lui à $34,34 \%$ et que le 
secondaire à 24,01\%. Les moyennes arithmétiques garantissent comme prouvé ci-haut la différence de moyennes entre les secteurs.

La comparaison de moyennes de secteurs à partir Fisher-Snedecor teste statistiquement ce résultat. L'article passe par les conditions suivantes :

- Ho : M1=M2=M3 : pas de différences de moyennes, ce qui veut dire que les secteurs économiques contribuent au même niveau dans la production du pays ;

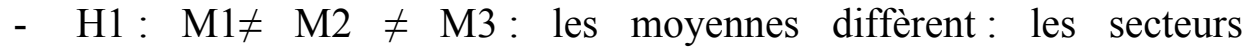
économiques contribuent différemment dans la production du pays.

On rejettera ici Ho si Fcal $>$ Ftab. Dans ce cas, le résultat fera étant d'une contribution différente des secteurs autrement dit un secteur contribue significativement que les autres et celui qui contribue plus pourra automatiquement profiter de cette économie d'échelle.

A partir des paramètres de tendance centrale et les littératures empiriques, on prévoit la différence des moyennes de secteurs et que le secteur primaire participe à une grande part que d'autres secteurs.

\section{DISCUSSION ET IMPLICATIONS MANAGERIALES}

Tableau 4 : Analyse de variances (Fisher Snedecor)

\begin{tabular}{|l|l|l|l|l|l|}
\hline $\begin{array}{l}\text { Source } \\
\text { variations }\end{array}$ & Somme des carrés & ddl & Moyenne des carrés & F & Probabilité \\
\hline Entre Groupes & 167954017135385,00 & 2 & 83977008567692,30 & 2,386474796 & 0,11107955 \\
\hline $\begin{array}{l}\text { A l'intérieur des } \\
\text { groupes }\end{array}$ & 950095611746031,00 & 27 & 35188726360964,10 & & \\
\cline { 1 - 4 } Total & 1118049628881420,00 & 29 & &
\end{tabular}

Source : Généré par Ms Excel à partir de nos calculs.

Avec une marge d'erreur de 0,05 et 2, 27 de ddl, le Fisher critique est 3,34 ; une valeur largement supérieure à 2,386474796. On accepte ici, l'hypothèse nulle selon laquelle il n'y a pas de différences de moyennes entre les secteurs économiques de la RDC avec la chance de se tromper jusqu'à un niveau supérieur de 0,11 . Dans ce cas, aucun secteur ne contribue pas plus que les autres statiquement. Les autorités pourront opter pour n'importe lequel dans l'application de l'économie d'échelle.

Plusieurs études qui ont fait l'objet de la littérature empirique ont montré que les secteurs économiques primaires et tertiaires contribuent plus dans les économies des pays. Quant à la RDC, la croissance est tirée par le secteur minier qui s'est accru de 10,5\% en moyenne entre 2010 et 2013 (Banque Mondiale 2014). Du secteur tertiaire, l'économie congolaise a affiché une croissance de $6,5 \%$ en 2011 contre 7,2\% en 2010, tirée par l'agriculture, les industries extraverties et le commerce (OCDE, 2012). Ces résultats sont corroborés par ceux obtenus dans cet article. Ainsi, les secteurs ont contribué, en moyenne, dans la production du pays à hauteur de $41,65 \%$ pour le secteur primaire, $34,34 \%$ pour le secteur tertiaire et à $24,01 \%$ pour le secteur secondaire (Tableau 3). 
Les résultats de cette étude montrent qu'en moyenne arithmétique, le secteur primaire contribue plus suivi du secteur tertiaire. On est d'avis avec Pierre \& Anna (2005) qui démontrent l'importance du capital humain ou physique à long terme. L'offre (capital, terre) a une importance par rapport aux facteurs relatifs à la demande (prix modélisés par les politiques internes et externes). L'exploitation de la terre relève du secteur primaire et celle du capital humain du secteur tertiaire. Le commerce a connu une expansion de $7 \%$ en 2011. Sa contribution à la croissance est passée à 1,4 point contre 0,99 en 2010. Il a tiré du bon comportement du secteur minier et de l'amélioration des infrastructures routières (OCDE, 2012). Cet article n'a pas pris en compte l'aspect de coût que va engendrer l'application d'une économie d'échelle tel que soulevé par Krugman \& al (2017): «le commerce international a souvent des effets importants sur la distribution des revenus au sein d'un pays. Il fait donc des perdants et des gagnants.

Cependant, la volonté politique pose problème dans la croissance économique. Il a été vu d'ailleurs que les institutions faibles du pays ont échoué à bâtir les fondations d'une économie résiliente et à absorber les chocs externes, exposant ainsi la société à des cycles de violence et d'appauvrissement (Banque Mondiale, 2018). La prise en compte d'implication du capital humain dans le secteur serait l'élément important dans ce cas de mutation de secteur.

Dans le cadre de la RDC, cet article suggère de bien adhérer aux conclusions telles qu'une croissance stable ou équilibrée exige à la fois le plein emploi et l'équilibre entre l'épargne et l'investissement (fil de rasoir). Elle nécessite aussi une combinaison optimale des facteurs travail et capital considérés complémentaires (Harrod, 1939) et (Domar, 1946).

Et surtout lorsqu'on tient compte de R. Solow et T. Swan (1956), qui ont parlé de modèle de Solow-Swan et considéré que les marchés sont en équilibre, les facteurs de production sont substituables et ont chacun un rendement marginal décroissant, mais la production a des rendements d'échelle constants.

Les résultats de ce travail ont pu démontrer le cas de la $\mathrm{RDC}$ où il y a absence de différences de moyennes entre les secteurs économiques avec une marge d'erreur supérieur de 0,11. Avec une marge d'erreur de 0,05 et 2, 27 de ddl, la valeur de Fisher critique est 3,34. Autrement dit, aucun secteur n'a contribué significativement que les autres. On peut donc appliquer une économie d'échelle dans un secteur de choix mais une attention particulière doit être prise en compte d'autant plus l'analyse n'a pas intégré d'information sur les facteurs de production d'ordre général et des facteurs spécifiques comme démontrent Krugman \& al. (2017) et Massassi \& al. (2018) que la richesse d'informations ancre bien une richesse de quantité et de qualité. 
Si on se contentait des paramètres de tendance centrale (moyennes arithmétiques) et de modèle Solow-Swan, on pouvait conseiller les autorités bancaires et politiques à exploiter plus le secteur primaire (Agricole et minier) accompagné de secteur tertiaire, ce qui rejoint la recommandation de la Banque Mondiale (2014) qui rappelle qu'investir dans ce secteur est essentiel pour faire reculer la pauvreté. Cette recommandation demande l'accompagnement de développement d'une collaboration gagant-gagant accompagnée d'une maîtrise de risques entre les secteurs primaires et tertiaires qui doivent travailler en étroite collaboration dans ce temps, si les collaborations entre pays telles qu'évoquées par Abrighach \& al. (2019) souffrent d'application.

\section{Conclusion}

Dans une situation de l'autarcie forcée par l'avènement de la Covid-19, les pays font face à des perturbations de leurs prévisions et sont soumis à des études approfondies pour s'adapter à une nouvelle configuration économique naturelle. Cet article a déterminé dans quel secteur économique la République Démocratique du Congo doit-il se spécialiser pour profiter de l'économie d'échelle interne. La méthode quantitative dans son approche statistique a permis de faire le test de comparaison des moyennes à travers la loi de F-Snedecor.

Les résultats font étant de l'absence de différence de moyennes de secteurs économiques, ce qui dit qu'aucun secteur ne contribue significativement à la production du pays. Pratiquement, les résultats invitent les autorités politiques et opérateurs économiques l'application de l'économie d'échelle dans un secteur de choix. Mais, si on se contentait des paramètres de tendance centrale (moyennes arithmétiques) et de modèle Solow-Swan, il serait conseillé aux autorités bancaires et politiques à beaucoup exploiter le secteur primaire (Agricole et minier) accompagné du secteur tertiaire. Cela permettra de faire reculer la pauvreté.

A travers la présente recherche, on peut noter la présence d'une limite sur la détermination des facteurs de production d'ordre général qui peuvent se déplacer entre les secteurs et des facteurs spécifiques. Ainsi, les prochaines étapes de notre recherche consisteront à déterminer le coût de la mutation d'un service à l'autre pour comparer le coût qui serait approprié pour la RDC dans la prise d'une décision relative à l'application d'une économie d'échelle pendant cette période de Covid-19, cela reste un pas qui permettra à répondre au besoin du moment. 


\section{Bibliographie}

Abrighach S. \& Meriouh Y. (2019). Usage des systèmes d'information interorganisationnels, agilité et performance de la chaine logistique. Vers un nouveau modèle de recherche, Europeen Scientific Journal, vol. 15, $N^{\circ} 10$, 332-358

Aghion P. and Howwitt P. (1992). A Model of Growth through Creative Destruction, in Econometrica, Vol. 60, $n^{\circ} 2,323-351$.

ANDS (2015). Note d'analyse des comptes nationaux provisoires 2013, semi-définitifs 29012 et définitifs 2011, Sénégal, 18.

Banque Centrale du Congo. (2019). Rapport Annuel.

Banque Mondiale. (2014). Rapport annuel, 67.

Banque Mondiale. (2018). République Démocratique du Congo. Diagnostic systématique pays: priorités de politique pour réduire la pauvreté et promouvoir la prospérité partagée dans un pays fragile sortant d'un conflit, Rapport $\mathrm{N}^{\circ} 112733-\mathrm{ZR}, 188$

Barro R. J. (1990), "Government Spending in a Simple Model of Endogenous Growth », Journal of Political Economy, 103-125.

Benoît C. (2020). "Les banques centrales pendant et après la pandémie de Covid-19, in Revue d'économie financière, $N^{\circ} 139-140,269-280$.

Bri (2020). «Annual Economic Report 2020 ». Banques des règlements internationaux.

Bonkena P.B. \& Roddy Miteu K.A. (2020). Performance des "Firmes Modèles" du Corridor de Monkoto dans la Province de la Tshuapa, République Démocratique du Congo, Europeen Scientific Journal, Vol. 16, $N^{\circ} 24,206-227$.

Cervantes-Godey D. \& Dewbre J. (2010). Importance économique de l'agriculture dans la lutte contre la pauvreté, Editions OCDE. doi: $10.1787 / 5 \mathrm{kmjiv} 40 / \mathrm{p} 5 \mathrm{~kg}-\mathrm{fr}, 29$.

Direction de l'analyse de la conjoncture industrielle. (Novembre 1996). La tertiairisation de l'économie du Québec ", Développement économique, innovation et exportation, Québec, 21.

Donald R. (2007). Les trois approches de MALTHUS pour résoudre le problème démographique, Cairn. Info, 253-280.

https://Deskeco.com consulté samedi 4 avril 2020 à $17 \mathrm{~h} 04$.

Kibala Kuma J. (décembre 2019). Modèle de croissance et politique économique en RDC : une analyse descriptive, CER-3/CREQ, working paper $n^{\circ} 004 / W P . C R E Q / 12-19,22$.

Krugman P., Obstfeld M. \& Melitz M. (2017). Economie Internationale-10 édition. Nouveaux Horizons, 751.

Mankiw G., Romer D. \& Wel D. (1992). A contribution to the empirics of Economic Growth, in Quartely Journal of Economics, $n^{\circ}$ 57, 407-437. 
Michel A. \& Sabrina K. (2020). Les innovations des banques centrales dans la crise de la Covid-19, in Révue d'économie Financière, $N^{\circ} 139-140,281-$ 291.

Mohamed A. M. \& Jaouad R. (2016). L'impact des pratiques qualité sur l'implication du personnel sous contrat flexible. Essai d'une Etude qualitative exploratoire auprès des entreprises du secteur automobile sur la région du Nord du Maroc, Europeen Scientific Journal, vol. 12, $N^{\circ} 25$, 199213.

Mssassi S. \& Assabane I. (2018). Risques et rôles en PME Exportatrice : la notion de veille stratégique, Europeen Scientific Journal, vol. 14, $N^{\circ} 34$, 268291.

Nicolas Meyer. (Mars 2011). Analyse de la variance: comparaison des plusieurs moyennes, Laboratoire de Bio statistique et informatique Médicale. Fac de Médicine de Strasbourg, 35.

OCDE. (2005). Les services et la croissance économiques: emploi, productivité et innovation, Réunion du Conseil de l'OCDE au ministériel 2005, 27.

OCDE. (2012). Perspectives économiques en Afrique 2012, BAfd, 15.

Pierre B. \& Anna L. (2005). Quel rôle joue l'agriculture dans la croissance et le développement ?, Revue Tiers Monde, 2005/3 n 183, 603 à 624.

Romer P. M. (1986). Increasing Returns and Long Run Growth, in Journal of Political Economy, Vol. 94, $n^{\circ} 5,1002-1037$.

Solow R.M. (1956). A contribution to the theory of Economic Growth, in Quartely Journal of Economics, Vol. 70, $n^{\circ}$ 1, 65-94.

Swan T.W. (1956). Economics of Growth and Capital Accumulation, in Economic Record, Vol. 32, 334-361.

Toure B. \& Macwilliam D. (2014). Le point de la situation économique au Bénin, gestion macroéconomique et budgétaire, Banque Mondiale, 30.

- www.brh.net/index.html 